\title{
Influencia del contenido de silicio y el tratamiento térmico en la resistencia al desgaste de fundiciones blancas al cromo en condiciones de rápida solidificación ${ }^{(\cdot)}$
}

\author{
L. Goyos*, A. Varela**, M. Verhaege***, A. García**, J. Mier** y M. Moors***
}

\begin{abstract}
Resumen
Se estudia la influencia del silicio y el tratamiento térmico sobre la microestructura y la resistencia al desgaste abrasivo y por fricción seca de una fundición con $3 \%$ y y 12 \% Cr, obtenida en condiciones de rápida solidificación. La rápida solidificación disminuye el volumen de carburos, la finura y dispersión de éstos aumentan con el incremento del silicio. Todas las muestras presentaron matrices perlíticas, variando en finura con el tratamiento. No se apreciaron productos de transformación intermedios. Las durezas tuvieron poca variación. Los tratamientos de austenización mostraron poca efectividad, con tendencia a desgastes más elevados que en estado bruto de colada y con tratamiento de mantenimiento. Los comportamientos ante la abrasión y la fricción seca en las condiciones ensayadas fueron similares. Se evidenció alta influencia de la morfología de los carburos en el desgaste abrasivo.
\end{abstract}

Palabras clave Fundiciones blancas al cromo; Tratamiento térmico; Desgaste abrasivo; Fricción seca.

\section{Influence of silicon content and heat treatment on wear resistance of white chromium cast irons under high speed solidification conditions}

\begin{abstract}
The influence of silicon content and heat treatment on microstructure, abrasive and dry friction wear resistance of a $3 \% \mathrm{C}, 12 \% \mathrm{Cr}$ cast iron, under fast solidification conditions is studied. The fast solidification condition diminishes the carbide volume and the silicon content increases their dispersion and finesses. All matrixes obtained were perlitics, whit different finesses. No intermediate transformation products were noticed. Hardness had little variation. Austenization treatment show little affectivity, with tendency to increase wear in reference to as cast and maintenance treatments. Behavior under dry friction and abrasive wear were similar under test conditions applied whit more influence of carbide morphology in the abrasive wear conditions.
\end{abstract}

Keywords

White chromium cast iron; Heat treatment; Abrasive wear; Dry friction.

\section{INTRODUCCIÓN}

Las fundiciones blancas al cromo constituyen un material de frecuente aplicación en la fabricación de diferentes elementos de la industria minera y de canteras $^{[1-5]}$ desde el pasado siglo. Su alta resistencia al desgaste, unida a una buena resistencia al impacto, las hace la selección obligada en muchas aplicaciones.

Una de las causas de la popularidad de estas fundiciones ha sido la posibilidad de obtención de dife- rentes estructuras en la matriz, sea directamente de colada o mediante diferentes tratamientos térmicos, aspectos que se vienen estudiando desde su introducción ${ }^{[5-8]}$.

Las fundiciones blancas al cromo presentan contenidos de cromo entre $12-30 \%$, con adiciones de otros elementos de aleación, comúnmente molibdeno y níquel, según consigna la especificación A532 de la ASTM. En estado bruto de colada la matriz es comúnmente perlítica ${ }^{[9-11]}$, aunque en función de las dimensiones de la pieza y los elementos de aleación

(•) Trabajo recibido el día 30 de enero de 2012 y aceptado en su forma final el día 22 de febrero de 2012.

* Grupo de Ingeniería de Materiales, Fac. de Ingeniería Mecánica, Instituto Superior Politécnico "José A. Echeverría", Calle 114 No. 11901, CP 19390, Marianao, Habana, Cuba.

** Grupo de Investigación de Ciencia e Ingeniería de Materiales, Universidad de La Coruña, Calle Mendizábal s/n, CP 15403,Ferrol, España.

*** Departamento de Ciencia e Ingeniería de Materiales, Universidad de Gante, Technologiepark 9, CP 9052, Zwijnaarde, Gante, Bélgica. 
aparecen otras estructuras. Es característica en estos materiales la presencia de carburos en cantidades importantes. El tratamiento térmico provoca, generalmente, la aparición de carburos secundarios distribuidos y con mayor o menor finura ${ }^{[3,11-13]}$.

En lo fundamental la adecuada combinación de propiedades se alcanza partiendo de una matriz resistente y una conveniente estructura de carburos, tanto en volumen como en su distribución y espacia-

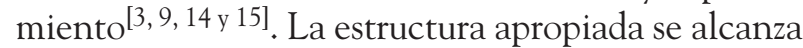
a través de una adecuada selección de la composición química y un tratamiento térmico que asegure una matriz y distribución de carburos acorde al trabajo de la pieza.

La estructura de la matriz ha sido objeto de par-

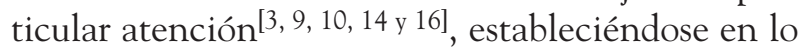
fundamental la relación entre ésta, el tipo de desgaste y los esfuerzos asociados al mismo.

La influencia de la orientación de los carburos primarios sobre la resistencia al desgaste ha sido señalada por Zum Ghar y Dogan ${ }^{[15}$ y 17]. Los trabajos de estos autores establecen que aparece un máximo de resistencia cuando los ejes principales de las dendritas de los carburos son paralelos a la superficie desgastada y orientados perpendicularmente a la acción abrasiva.

Bajo la acción de impactos repetidos, por demás frecuentes en las aplicaciones corrientes de estas fundiciones, resulta posible la aparición de la fatiga superficial y el descamado de la superficie. Este es un fenómeno corriente en las bolas para molienda y se asocia con la presencia y posterior transformación por impacto de la austenita residual.

Diferentes elementos de aleación han sido usados para garantizar la estructura y templabilidad deseadas en estos materiales, comúnmente el molibdeno, así como el níquel y el cobre ${ }^{[2,5,9,18 \text { y 19]. }}$.

La presencia del silicio en la composición de estas fundiciones se ha asociado con la disminución en dureza y resistencia al desgaste debido a la aparición de perlita en la estructura ${ }^{[2,5}$ y 19]. Sin embargo, la influencia del silicio puede permitir una adecuada regulación de la precipitación de carburos y por ende de las propiedades finales de la pieza, lo que explica que en los últimos años se haya concedido una nueva atención al papel de este elemento en las fundiciones al cromo.

Wei ${ }^{[20]}$ señala las ventajas de disminuir el contenido de cromo en la aleación y adicionar silicio como una alternativa para el uso de estas fundiciones en aplicaciones de desgaste e impacto repetido. En este caso el autor utiliza una matriz perlítica, obtenida directamente de colada, atribuyendo la elevada resistencia a la fatiga de impacto y las buenas propiedades de resistencia al desgaste a la uniformidad de la estructura, la mejor morfología de los carburos y la ausencia de carburos secundarios que actúen como iniciadores de grietas.
Bedolla y Rainforth ${ }^{[21]}$ trabajaron sobre aleaciones con $17 \%$ de cromo, con molibdeno y níquel alrededor del $2 \%$, haciendo variar el contenido de silicio hasta el $5 \%$. Estas aleaciones fueron coladas en moldes metálicos y señalan que el incremento del silicio refina la estructura dendrítica de los hierros al cromo a la vez que incrementa el volumen de carburos. Se destaca el papel de la velocidad de solidificación en este efecto. Estos autores determinaron un máximo en la resistencia al desgaste de altos esfuerzos por fricción seca de la aleación para contenidos de silicio alrededor del $2 \%$. No reportan diferencias significativas entre el comportamiento de las aleaciones en estado de colada y tratadas térmicamente, lo que atribuyen a la transformación de la austenita presente en las aleaciones en estado bruto de colada. Destacan el papel de la morfología y propiedades de los carburos en el comportamiento de la aleación.

Es de destacar que la composición con el máximo de resistencia al desgaste determinado por estos autores coincide prácticamente con la composición eutéctica.

El comportamiento en condiciones de tratamiento térmico de estas fundiciones ha sido estudiado por diferentes autores ${ }^{[2,5-8,11,19,22-25]}$. En sentido general las fundiciones de alto cromo han sido usadas en estado de colada, con tratamientos térmicos a temperaturas por encima de la temperatura de transformación y con tratamientos por debajo de la misma. Se destaca en estas aleaciones la necesidad de disminuir el contenido de carbono en la austenita por medio de la precipitación de carburos con el fin de lograr

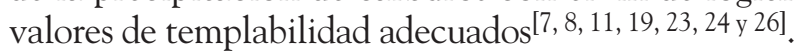
Este tratamiento, a temperaturas generalmente superiores a $\operatorname{los} 950^{\circ} \mathrm{C}$, trae aparejado el aumento del tiempo de inicio de las transformaciones en condi-

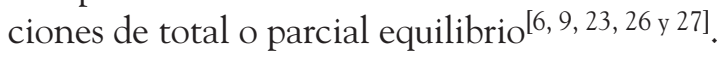

Los tratamientos subcríticos se mueven en un amplio intervalo de temperaturas, en función de la estructura perseguida. Estos tratamientos resultan atractivos por su relativa economía y las propiedades

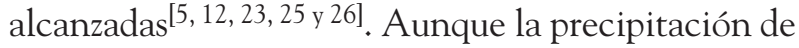
carburos secundarios no es común en estos tratamientos, los mismos han sido documentados en tratamientos a temperaturas cercanas a la de transformación ${ }^{[26]}$. Resultan de particular interés los trabajos de Wei ${ }^{[20]}$ y la comparación entre la resistencia al impacto repetido de la perlita y la martensita.

La aplicación de tratamientos isotérmicos para la obtención de estructuras bainíticas ha sido poco abordada en las fundiciones al cromo, a pesar de sus aplicaciones frecuentes en otras aleaciones cuando se busca mejorar la tenacidad de impacto. La presencia de austenita retenida y la potencial transformación posterior de ésta hacen que esta opción sea poco evaluada en estos materiales y en general evitada ${ }^{[25]}$, dada la presencia de impactos repetidos en muchas 
de las aplicaciones habituales. No obstante la bainita en sí misma puede ser una estructura ventajosa por sus propiedades, lo que hace su estudio interesante. $\mathrm{Hou}^{[22]}$ señala la positiva influencia que ejerce sobre la resistencia al impacto el disminuir el contenido de austenita, usando para ello temperaturas de tratamiento cercanas al inicio de la transformación martensítica. La temperatura de tratamiento es, en buena medida, responsable de la estructura y las propiedades finales obtenidas ${ }^{[12]}$. Resulta también de interés el comportamiento de la transformación bainítica ante la presencia del silicio, así como la influencia del tratamiento térmico sobre la disminución de la austenita residual, según enuncia Santofimia ${ }^{[28]}$.

De acuerdo con los trabajos de Maratray ${ }^{[11]}$ la bainita aparece en aleaciones con relaciones $\mathrm{Cr} / \mathrm{C}$ inferiores a 4. Aun así se requiere de tiempos de mantenimiento isotérmico del orden de 6 h o más para lograr un porcentaje de transformación importante. La adición de silicio a la aleación puede resultar efectiva en la disminución de los tiempos de tratamiento ${ }^{[28]}$.

El amplio abanico de propiedades que exhiben las fundiciones al cromo, en dependencia de su composición y tratamientos, constituye un campo prometedor para diferentes aplicaciones. El uso del silicio, conjuntamente con otros elementos de aleación, que se reporta en la literatura consultada, así como las diferentes condiciones de tratamiento y ensayo de estos hierros por los diferentes autores, abren un horizonte prometedor, pero no determinan con exactitud diferentes aspectos. La efectividad de la adición del silicio en la disminución del tiempo de tratamiento para la obtención de bainita es un ejemplo. Adicionalmente hay aspectos cuya influencia no está documentada, como es el efecto del tratamiento subcrítico sobre las estructuras obtenidas mediante solidificación rápida.

De forma similar la influencia de estos aspectos sobre la resistencia al desgaste no aparece referida.

\section{PROCEDIMIENTO EXPERIMENTAL}

En el presente trabajo se partió de una aleación al cromo 3-12, solidificada en condiciones de rápido enfria- miento, con el fin de estudiar el efecto de la adición de silicio y la aplicación de diferentes tratamientos térmicos sobre su estructura y resistencia ante el desgaste.

La aleación seleccionada, con composición ligeramente hipoeutéctica, presenta aproximadamente 0,6 \% de silicio. Esta composición sitúa a la aleación base en el campo correspondiente a la formación de los carburos del tipo $\mathrm{M}_{7} \mathrm{C}_{3}$ en condiciones estables, según el diagrama de Jackson ${ }^{[29]}$. La relación $\mathrm{Cr} / \mathrm{C}=4$ de esta aleación garantiza la posibilidad de aparición de la bainita sin la adición de otros elementos de aleación ${ }^{[30]}$. Por otro lado los bajos contenidos de carbono y cromo evitan una excesiva presencia de carburos.

Una segunda aleación se obtuvo adicionando silicio a la aleación base hasta un 2,4 \%, manteniéndose la composición prácticamente eutéctica.

Se utilizó para la fusión arrabio sintético, ferrocromo de bajo carbono y ferrosilicio en las proporciones adecuadas. La fusión se realizó en un horno de alta frecuencia con crisol de $5 \mathrm{~kg}$. Las composiciones obtenidas se muestran en la tabla I.

La colada se realizó a temperatura de $1.380^{\circ} \mathrm{C}$ y el metal fue vertido en lingoteras metálicas rectangulares con sección $15 \times 8 \mathrm{~mm}$ y $250 \mathrm{~mm}$ de longitud. A partir de estas placas se elaboraron las probetas de dimensiones $15 \times 15 \times 5$.

El uso de lingoteras y el vertido en placas de reducido espesor permite asegurar una velocidad de enfriamiento y solidificación considerable.

Esta forma de obtención del semiproducto y la posterior elaboración de las probetas garantiza que la superficie de ensayo de las mismas sea perpendicular a la dirección preferente de evacuación del calor. De esta forma todos los ensayos se realizarán sobre una superficie transversal a los ejes principales de las dendritas de los carburos primarios. Con esto se garantiza una total uniformidad en las características de las superficies de ensayo, aunque esta orientación resulta la más desventajosa para la resistencia al desgaste.

Los tratamientos aplicados persiguieron determinar el comportamiento de las aleaciones ante tratamientos en la zona de transformación bainítica. Fueron aplicados dos tipos de tratamientos diferentes a temperaturas superiores e inferiores a la temperatura crítica de transformación.

Tabla I. Composición química de las aleaciones

Table I. Chemical composition of alloys

\begin{tabular}{lllllll}
\hline Aleación & C [\%] & Cr [\%] & Si [\%] & Mn [\%] & S [\%] & P [\%] \\
\hline Alto Si (A) & 3,02 & 12,02 & 2,4 & 0,51 & 0,002 & 0,002 \\
Bajo Si (B) & 3,05 & 12,10 & 0,63 & 0,5 & 0,002 & 0,002 \\
\hline
\end{tabular}


El tratamiento por encima de la temperatura crítica consistió en la austenización y posterior desestabilización de la austenita mediante mantenimiento a $970{ }^{\circ} \mathrm{C}$ durante $1 \mathrm{~h}$, seguida de enfriamiento al aire y mantenimiento isotérmico a $250^{\circ} \mathrm{C}$ por tiempos de 45,90 , 180 y 360 min en el horno. La temperatura de desestabilización se seleccionó teniendo en cuenta la composición química obtenida y el diagrama de transformación correspondiente según los trabajos de Maratray ${ }^{[11]}$ y Kulmburg ${ }^{[11]}$. Para la selección de la temperatura de tratamiento isotérmico se tuvo en cuenta la posición del campo bainítico y la temperatura de inicio de la transformación martensítica ${ }^{[31]}$. Para la selección de los tiem- pos de mantenimiento a la temperatura de transformación se tuvo en cuenta la templabilidad bainítica de

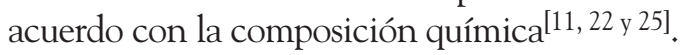

El tratamiento subcrítico aplicado consistió en un mantenimiento isotérmico a $250^{\circ} \mathrm{C}$, directamente desde el estado de colada, por tiempos de 180 y $360 \mathrm{~min}$. Para la selección de la temperatura y tiempos de tratamiento se siguió el mismo criterio que para los tratamientos por encima de la temperatura crítica.

Con fines de comparación se utilizaron las aleaciones en estado bruto de colada. Los tratamientos, identificación de las muestras y propiedades obtenidas aparecen en la tabla II.

Tabla II. Designación, tratamiento térmico y algunas propiedades determinadas en las muestras Table II. Designation, heat treatment and several properties of specimens

\begin{tabular}{|c|c|c|c|c|c|c|c|c|}
\hline Código & Aleación & $\begin{array}{l}\text { Tiempo } \\
\text { a } 970^{\circ} \mathrm{C}\end{array}$ & $\begin{array}{c}\text { Tiempo a } \\
250^{\circ} \mathrm{C} \text { [min] }\end{array}$ & $\begin{array}{l}\text { Dureza } \\
\text { [HRc] }\end{array}$ & $\begin{array}{c}\text { Austenita } \\
{[\%]}\end{array}$ & $\begin{array}{l}\text { Cromo en } \\
\text { matriz [\%] }\end{array}$ & $\begin{array}{l}\text { Cromo en } \\
\text { carburos [\%] }\end{array}$ & $\begin{array}{c}\text { Fuerza de } \\
\text { fricción media [N] }\end{array}$ \\
\hline $\bar{A}$ & $\begin{array}{l}\text { Alto } \\
\text { Si }\end{array}$ & - & & 55 & 0 & 7,48 & 39,76 & $\begin{array}{c}11,38 \\
\pm 1,942\end{array}$ \\
\hline B & $\begin{array}{l}\text { Bajo } \\
\mathrm{Si}\end{array}$ & - & & 55 & 0 & 8,99 & 41,42 & $\begin{array}{c}12,82 \\
\pm 1,845\end{array}$ \\
\hline AT45 & $\begin{array}{l}\text { Alto } \\
\text { Si }\end{array}$ & $1 \mathrm{~h}$ & 45 & 52 & 0 & & & $\begin{array}{c}14,54 \\
\pm 1,142 \\
\end{array}$ \\
\hline BT45 & $\begin{array}{l}\text { Bajo } \\
\mathrm{Si}\end{array}$ & $1 \mathrm{~h}$ & 45 & 53 & 0 & & & $\begin{array}{r}13,55 \\
\pm 1,681\end{array}$ \\
\hline AT90 & $\begin{array}{l}\text { Alto } \\
\mathrm{Si}\end{array}$ & $1 \mathrm{~h}$ & 90 & 52 & 0 & & & $\begin{array}{c}12,67 \\
\pm 1,372\end{array}$ \\
\hline ВT90 & $\begin{array}{l}\text { Bajo } \\
\mathrm{Si}\end{array}$ & $1 \mathrm{~h}$ & 90 & 53 & 6,7 & & & $\begin{array}{c}14,07 \\
\pm 1,360\end{array}$ \\
\hline AT180 & $\begin{array}{l}\text { Alto } \\
\text { Si }\end{array}$ & $1 \mathrm{~h}$ & 180 & 52 & 4 & & & $\begin{array}{r}13,05 \\
\pm 1,718 \\
\end{array}$ \\
\hline BT180 & $\begin{array}{l}\text { Bajo } \\
\mathrm{Si}\end{array}$ & $1 \mathrm{~h}$ & 180 & 51 & 10 & & & $\begin{array}{c}12,69 \\
\pm 2.072\end{array}$ \\
\hline AT360 & $\begin{array}{l}\text { Alto } \\
\mathrm{Si}\end{array}$ & $1 \mathrm{~h}$ & 360 & 52 & 0 & 6,78 & & $\begin{array}{r}12,62 \\
\pm 1,598 \\
\end{array}$ \\
\hline ВT360 & $\begin{array}{l}\text { Bajo } \\
\mathrm{Si}\end{array}$ & $1 \mathrm{~h}$ & 360 & 52 & 7,3 & 7,5 & & $\begin{array}{c}12,65 \\
\pm 1,240\end{array}$ \\
\hline AM180 & $\begin{array}{l}\text { Alto } \\
\text { Si }\end{array}$ & - & 180 & 55 & 0 & & & $\begin{array}{c}11,38 \\
\pm 1,491\end{array}$ \\
\hline BM180 & $\begin{array}{l}\text { Bajo } \\
\mathrm{Si}\end{array}$ & - & 180 & 56 & 0 & & & $\begin{array}{c}13.58 \\
\pm 2,088\end{array}$ \\
\hline AM360 & $\begin{array}{l}\text { Alto } \\
\mathrm{Si}\end{array}$ & - & 360 & 55 & 0 & 7,15 & & $\begin{array}{r}14,75 \\
\pm 1,256 \\
\end{array}$ \\
\hline BM360 & $\begin{array}{l}\text { Bajo } \\
\text { Si }\end{array}$ & - & 360 & 56 & 0 & 8,39 & & $\begin{array}{r}12,89 \\
\pm 1,807\end{array}$ \\
\hline
\end{tabular}


Las muestras fueron rectificadas después del tratamiento térmico para alcanzar una rugosidad superficial uniforme y correspondiente a 0,8 Ra aproximadamente.

Las muestras fueron sometidas a ensayos de dureza Rockwell C, con carga de 1.470 N. Se realizó la observación metalográfica por medio de microscopía óptica y electrónica de barrido. La presencia de los elementos de aleación en matriz y carburos fue determinada mediante difractometría (EDAX ZAF). La presencia de austenita en las muestras se determinó por difracción de rayos X mediante emisión MoKa $(30$ kV,25 mA) y para su cuantificación se siguió la ASTM St E975-84.

Para la cuantificación del volumen de carburos se siguió la metódica propuesta por Maratray ${ }^{[11]}$, realizando la evaluación de áreas por medio del análisis, mediante una aplicación al efecto, de las imágenes digitales adquiridas.

Se determinó el comportamiento de ambas aleaciones bajo los diferentes tratamientos en condiciones de desgaste abrasivo y fricción seca.

Los ensayos de desgaste abrasivo se efectuaron en un tribómetro tipo espiga sobre disco, en el cual se sustituyó la espiga por la muestra rectangular a ensayar, colocándola con la mayor superficie contra el disco. El ensayo se desarrolló bajo carga de $30 \mathrm{~N}$, contra un disco abrasivo de corindón grano 80 , durante un recorrido de $500 \mathrm{~m}$. La magnitud de la capa desgastada se evaluó por medio de la disminución de la diagonal de las indentaciones piramidales realizadas en los puntos extremos y en el centro de la muestra. Se evaluaron tres probetas de cada muestra utilizando finalmente el promedio de las mediciones fiables.
Los ensayos de desgaste por fricción seca se realizaron en un tribómetro tipo bola sobre disco (Microtest). En este caso la bola consiste en una esfera pulida de carburo de wolframio con diámetro de $4 \mathrm{~mm}$. Dicha bola actúa sobre la muestra rectificada en un circuito circular de $8 \mathrm{~mm}$ de diámetro bajo carga de $20 \mathrm{~N}$. El recorrido total fue de $2 \mathrm{~km}$. La evaluación del desgaste se efectuó por el método gravimétrico utilizando el promedio de cinco mediciones de peso fiables. Para la evaluación del error general del método se utilizó el error medio determinado en ensayos anteriores del equipo ${ }^{[32]}$. Adicionalmente se evaluó la fuerza de fricción presente en el ensayo mediante una celda de carga acoplada al brazo de sostén del soporte de la bola. Estos valores son registrados en tiempo real mediante una aplicación al efecto, al igual que las revoluciones.

\section{ANÁLISIS DE LOS RESULTADOS}

\subsection{Microestructura}

El análisis de las muestras con microscopía óptica evidencia diferencias morfológicas entre la aleación con bajo silicio y la aleación con alto silicio (Fig. 1). Ambas aleaciones muestran carburos pequeños y distribuidos en un patrón dendrítico típico de las aleaciones hipoeutécticas. La aleación con alto silicio muestra carburos más pequeños, redondeados y menos espaciados que la aleación con bajo silicio.

El volumen de carburos se comporta también de forma similar, arrojando un valor de 24,8 $\pm 2,04 \%$ para la aleación con alto silicio mientras que la aleación con bajo silicio muestra un 19,84 $\pm 3,76 \%$.

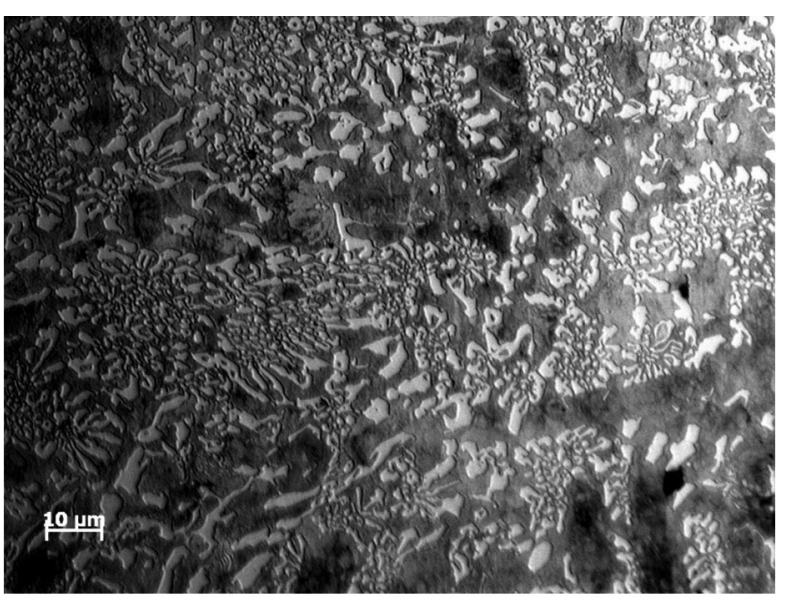

(A)

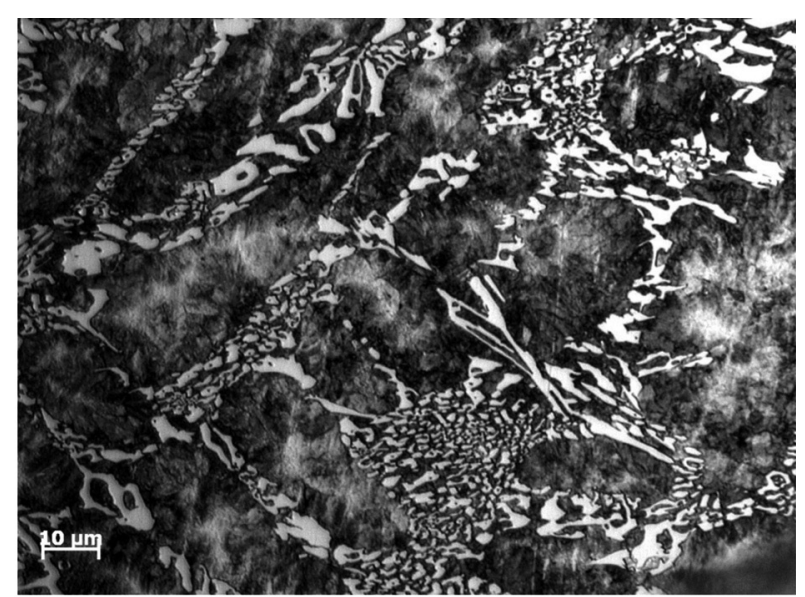

(B)

Figura 1. Aleación con alto silicio (A) y bajo silicio (B), en estado bruto de colada.

Figure 1. High $(A)$ and low $(B)$ silicon content alloys, as cast. 
Estos valores son aproximadamente un $5 \%$ inferiores a los resultados que arroja el cálculo de la fracción de carburos para condiciones de enfriamiento más moderadas, según la expresión de Maratray ${ }^{[30]}$. Este comportamiento es más acusado que el reportado por Bedolla ${ }^{[21]}$ en condiciones de colada en coquilla. Esta diferencia es debida al efecto que sobre la difusión de los elementos formadores de carburos ejercen las severas condiciones de enfriamiento prevalecientes en la obtención de las muestras, siendo más rigurosas que las condiciones establecidas en el trabajo referido.

La aleación con alto silicio presenta un volumen de carburos mayor que aquélla con silicio bajo, coincidiendo con lo citado por Bedolla ${ }^{[21]}$. La acción del silicio se manifiesta a través de la disminución de la actividad y solubilidad del carbono en la red, a la par que puede disminuir parcialmente las tensiones generadas en dicha red por la presencia de los átomos de cromo. No obstante la diferencia deter- minada entre ambas aleaciones no resulta mayor que la variabilidad de la medición, limitando su fiabilidad.

Ambas aleaciones muestran en el estado bruto de colada una matriz con un conglomerado perlítico muy fino, como se observa en la figura 2.

No hay evidencias de la aparición de martensita o productos intermedios en las aleaciones en estado bruto de colada. No se advierte acicularidad de la estructura, salvo en muy aisladas zonas de la aleación con bajo silicio. En esta misma aleación la estructura laminar presenta ocasionalmente diferentes orientaciones en pequeñas distancias.

Tampoco hay presencia de austenita, corroborado por los resultados de la difractometría. La dureza de ambas aleaciones en estado de colada es alta y prácticamente la misma para ambos casos.

El tratamiento aplicado de austenización y mantenimiento ulterior a $250^{\circ} \mathrm{C}$ por diferentes tiempos no provocó cambios substanciales en la estructura de

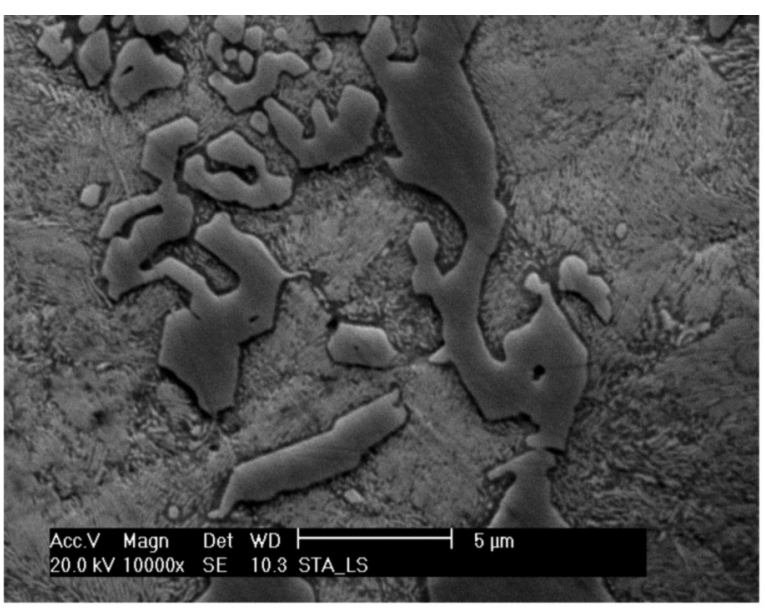

(A1)

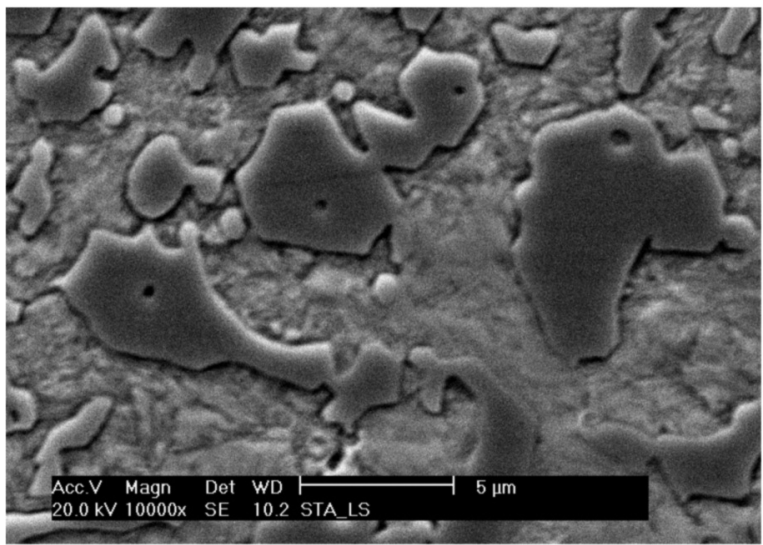

(B1)

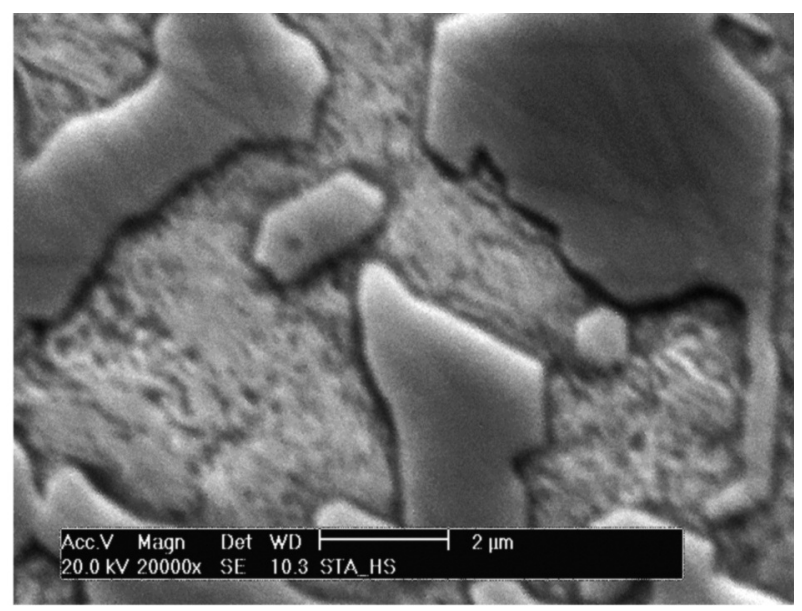

(A2)

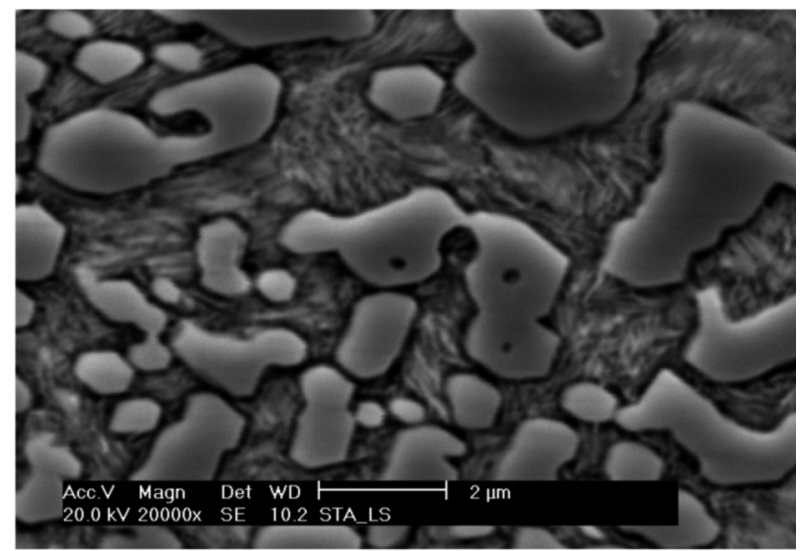

(B2)

Figura 2. Microestructura de las aleaciones con alto $\left(A_{1-2}\right)$ y bajo $\left(B_{1-2}\right)$ silicio en estado bruto de colada.

Figure 2. Microstructure of high $\left(A_{1-2}\right)$ and low silicon $\left(B_{1-2}\right)$ alloys, as cast. 
la matriz en ninguna de las aleaciones. Los diferentes tratamientos aplicados han conducido, en todos los casos, a una estructura perlítica muy fina y sin orientación preferente.

Aparece austenita en pequeños volúmenes en algunos casos, mayormente en la aleación con bajo silicio, como se muestra en la tabla II. El máximo de contenido de austenita aparece para el mantenimiento posterior durante $180 \mathrm{~min}$. Se hace evidente también la precipitación de carburos secundarios, con presencia aparentemente algo mayor en la aleación con alto silicio, de acuerdo con la observación (Fig. 3).

En la figura 4 se muestra la estructura de ambas aleaciones tras ser sometidas al tratamiento subcrítico (mantenimiento a $250{ }^{\circ} \mathrm{C}$ sin austenización previa).

La diferencia fundamental con las estructuras previamente austenizadas es la ausencia de carburos secundarios. En estas muestras se mantiene la estructura de productos perlíticos muy finos. La difractometría no arrojó presencia de austenita en estos casos.

La distribución del cromo en la matriz muestra valores algo superiores a los reportados por Maratray ${ }^{[30]}$ para aleaciones similares coladas en arena. La aleación con bajo silicio presenta 8,99 \% de cromo en matriz, mientras que Maratray reporta valores cerca del $7 \%$ para relaciones $\mathrm{Cr} / \mathrm{C}$ semejantes. Por su parte la aleación con 2,4 \% de silicio muestra valores de cromo en matriz similares a los de la aleación pobre en silicio. Este comportamiento de los valores de cromo en la matriz se corresponde con los menores volúmenes de carburos presentes en estas aleaciones debido al rápido enfriamiento.

En las muestras con tratamiento subcrítico se aprecia una muy pequeña disminución del cromo en matriz al compararlas con la aleación bruta de colada (aproximadamente 0,5\%) mientras que las austenizadas muestran un empobrecimiento mayor (alrededor del $1 \%)$.

Los carburos eutécticos presentes en las aleaciones se corresponden con carburos eutécticos de cromo y hierro, presentando los correspondientes a las aleaciones con bajo silicio un contenido de cromo ligeramente superior al de las aleaciones altas en silicio. Ambas aleaciones presentan valores comparables con los reportados por Maratray ${ }^{[30]}$.

Las aleaciones en estado bruto de colada no presentan austenita en su estructura. Las muestras con tratamiento de mantenimiento sin austenización previa tampoco manifiestan presencia de austenita. Tan solo las muestras con austenización presentan austenita en algunos casos. Los máximos en cada caso se corresponden con el tiempo de $3 \mathrm{~h}$, mientras que se aprecia un efecto marcado de la presencia del silicio en la disminución del volumen de austenita residual presente, lo que se corresponde con la influencia del silicio referida en la literatura.

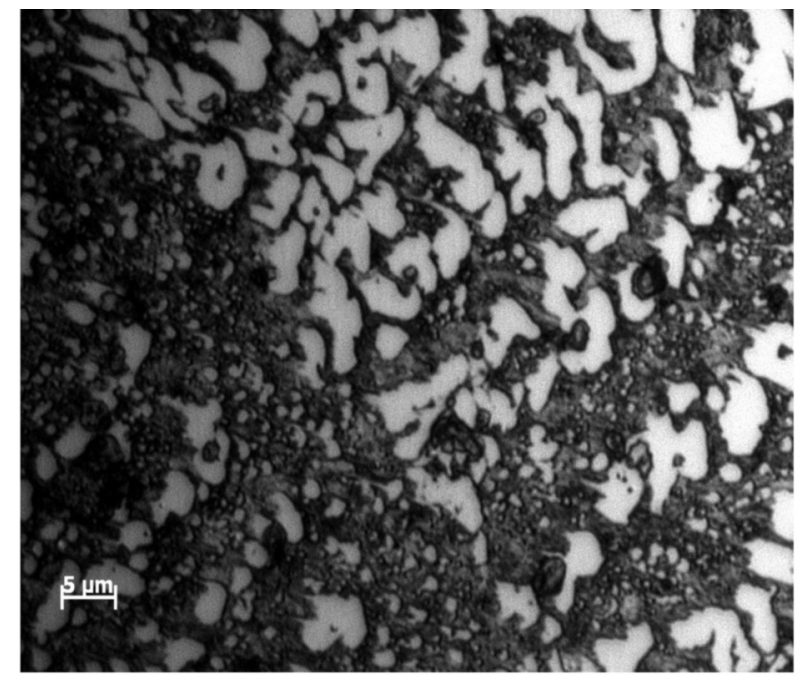

(A)

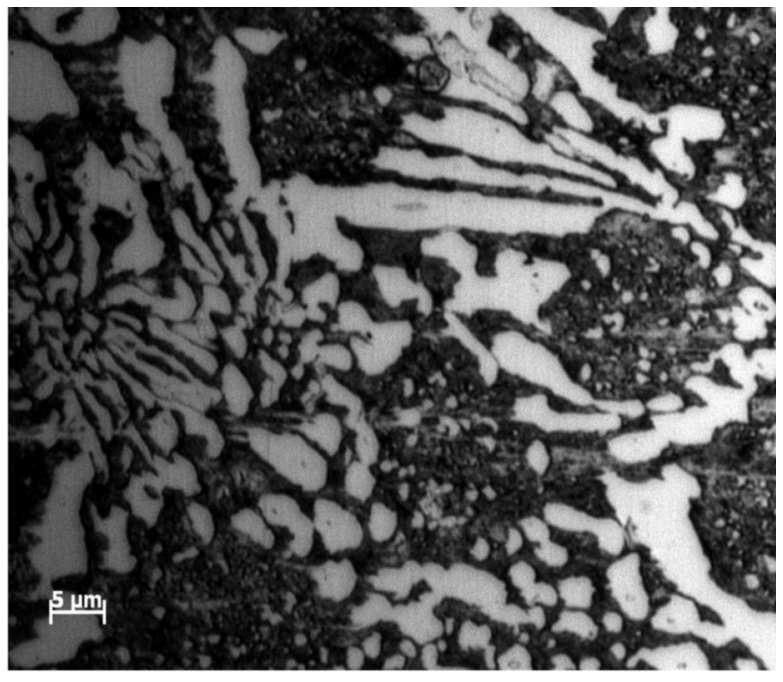

(B)

Figura 3. Microestructura de las aleaciones con alto $(A)$ y bajo $(B)$ silicio sometidas a austenización y posterior tratamiento a $250{ }^{\circ} \mathrm{C}$ durante $360 \mathrm{~min}$.

Figure 3. Microstructure of high (A) and low (B) silicon alloys, treated with austenization and holding at $250{ }^{\circ} \mathrm{C}$ for $360 \mathrm{~min}$. 


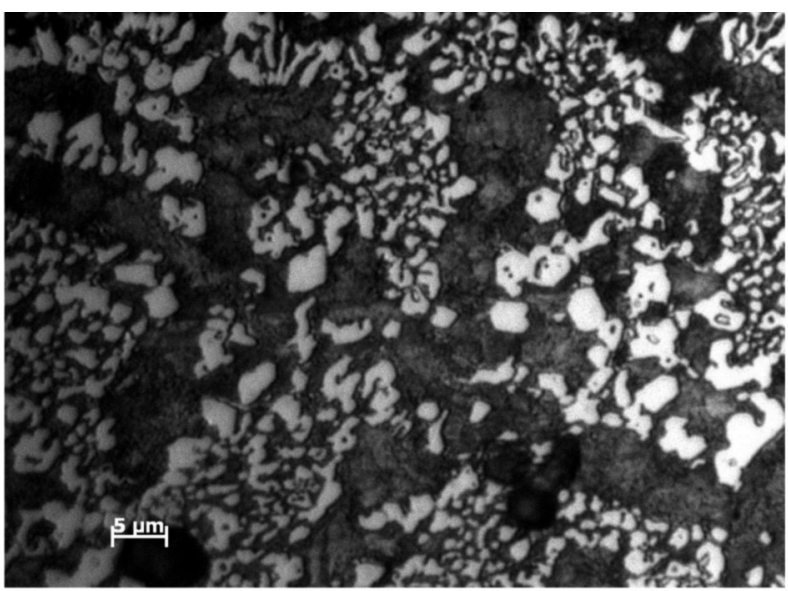

(A1)

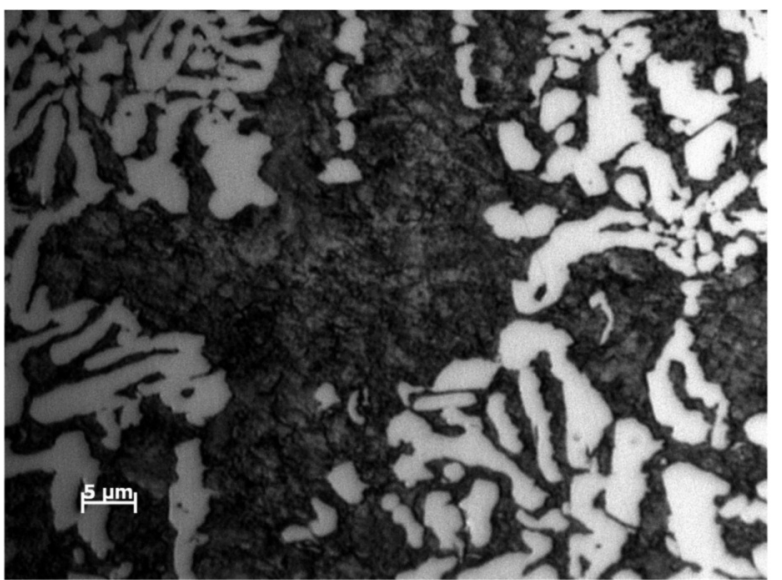

(B1)

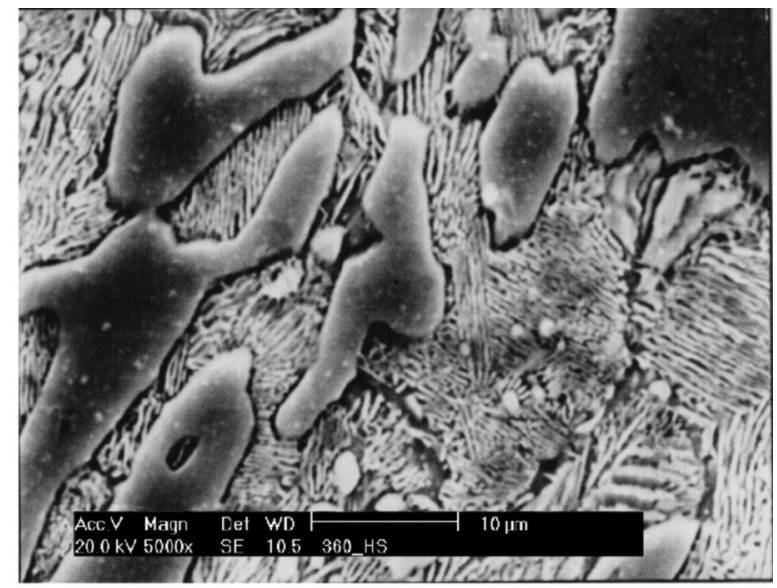

(A2)

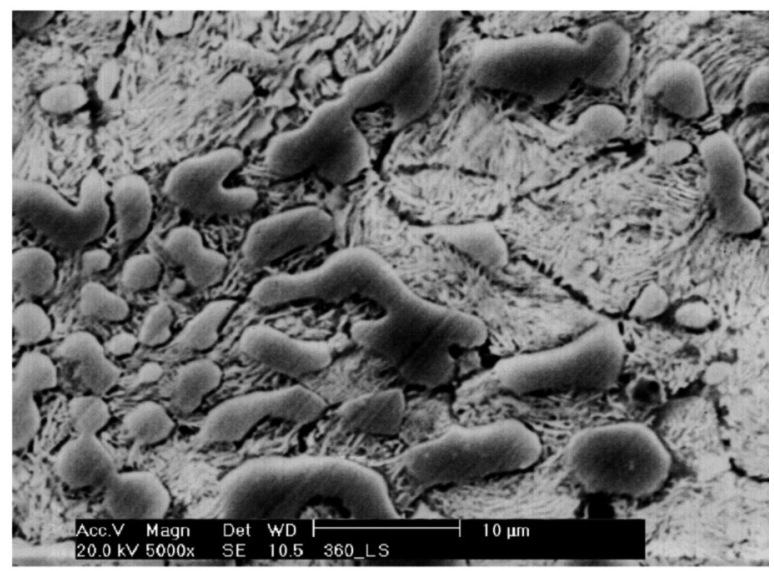

(B2)

Figura 4. Microestructura de las aleaciones con alto $\left(A_{1-2}\right)$ y bajo $\left(B_{1-2}\right)$ silicio sometidas a tratamiento subcrítico a $250^{\circ} \mathrm{C}$ durante $360 \mathrm{~min}$.

Figure 4. Microstructure of high $\left(A_{1-2}\right)$ and low $\left(B_{1-2}\right)$ silicon alloys, subcritically treated at $250{ }^{\circ} \mathrm{C}$ during $360 \mathrm{~min}$.

\subsection{Dureza}

Como se aprecia en la tabla II, en el comportamiento de la dureza de estas aleaciones la presencia del silicio no muestra tener un efecto importante. Ambas aleaciones presentan durezas comparables por lo que no se aprecia el efecto endurecedor del silicio sobre la ferrita para los porcentajes estudiados, en condiciones de enfriamiento rápido.

Las muestras con tratamiento de austenización, independientemente de los parámetros del tratamiento posterior, presentan durezas ligeramente menores que las aleaciones sin tratamiento o con tratamiento exclusivamente de mantenimiento. Esto permite concluir que la estructura tensionada obtenida por la colada en condiciones de rápido enfriamiento brinda durezas que son favorablemente comparables con las estructuras tratadas, sin diferencias notables al variar el contenido de silicio de la aleación o el tiempo de tratamiento. De hecho, ninguno de los tratamientos se justifica desde el punto de vista de la dureza.

\subsection{Desgaste abrasivo}

En la figura 5 se pone de manifiesto el comportamiento de las muestras ante el desgaste por abrasión.

En el caso del desgaste abrasivo los diferentes tratamientos aplicados no logran diferencias significativas en el comportamiento de esta propiedad para la mayoría de los casos. Por el contrario, la mayoría de los tratamientos presentan valores de desgaste superiores a los valores de las aleaciones en estado bruto de colada. El contenido de silicio presente, por 


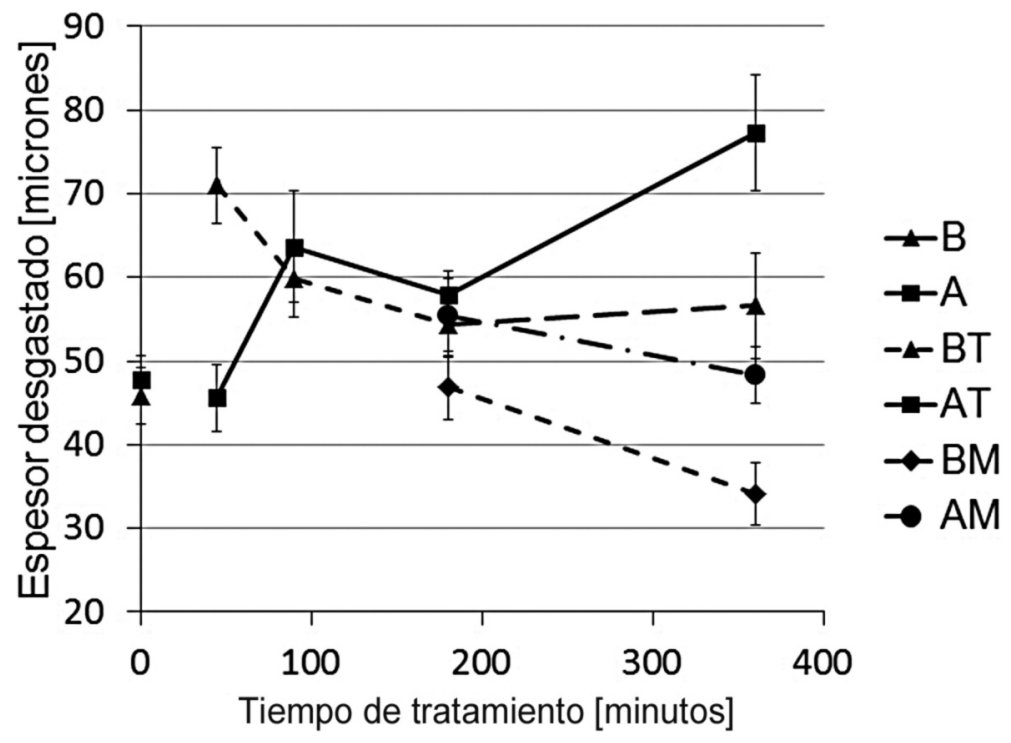

Figura 5. Comportamiento de las muestras bajo condiciones de desgaste abrasivo.

Figure 5. Wear of specimens under abrasion.

su parte, no ejerce influencia sobre la resistencia al desgaste.

Se destacan los resultados de la aleación con bajo silicio y tratamiento subcrítico. Los resultados más deficientes se corresponden con la aleación de alto contenido de silicio con tratamiento de austenización y mantenimiento durante $6 \mathrm{~h}$ a $250^{\circ} \mathrm{C}$.

En general ambas aleaciones presentan un buen comportamiento en estado bruto de colada y con tratamiento subcrítico, lo que responde también a las mayores durezas alcanzadas y a la estructura más resistente lograda por la rápida solidificación. Este comportamiento se compara favorablemente con los resultados obtenidos mediante la aplicación del tratamiento de transformación llevado a cabo. Para el tratamiento subcrítico se aprecia que con el aumento del tiempo de mantenimiento disminuye la pérdida de peso, lo que es posible relacionar con una mayor tenacidad de la matriz, debida a la relajación de tensiones que provoca el tratamiento.

El cambio estructural logrado mediante el tratamiento de austenización y mantenimiento no resultó efectivo en la mejora de la resistencia al desgaste abrasivo, a pesar de la precipitación de carburos secundarios para los tiempos medio y largo. Esto muestra que dichos carburos secundarios no representan una influencia fundamental en la elevación de la resistencia al desgaste abrasivo de estas aleaciones. El efecto de los carburos primarios resulta magnificado en este caso debido a la dis- tribución de los mismos. Como consecuencia del rápido enfriamiento las estructuras de ambas aleaciones presentan una distribución muy uniforme de los carburos primarios, sin grandes áreas expuestas de matriz que pudieran sufrir los efectos del desgaste, en función de las dimensiones del grano abrasivo.

La influencia de la presencia de austenita en las muestras sobre el comportamiento de las mismas ante el desgaste abrasivo resulta ligeramente favorable. En la aleación con bajo silicio, donde se presentaron los mayores volúmenes de austenita, el comportamiento del desgaste obtenido se corresponde en buena medida con la evolución de esta fase. En la aleación con alto silicio esta tendencia está presente también, aunque se hace notable un valor mínimo de desgaste para el tratamiento durante $45 \mathrm{~min}$, el cual no presenta una justificación estructural.

\subsection{Fricción seca}

El comportamiento de las muestras ante la fricción seca se muestra en la figura 6 .

En este caso se aprecia la tendencia a menores valores de desgaste en las muestras con tratamiento subcrítico, las cuales presentan los mayores valores de dureza; sin embargo esta tendencia no es tan marcada como en el caso del desgaste abrasivo. Se mantiene la tendencia a la disminución del desgaste con el aumento del tiempo de tratamiento debido al 


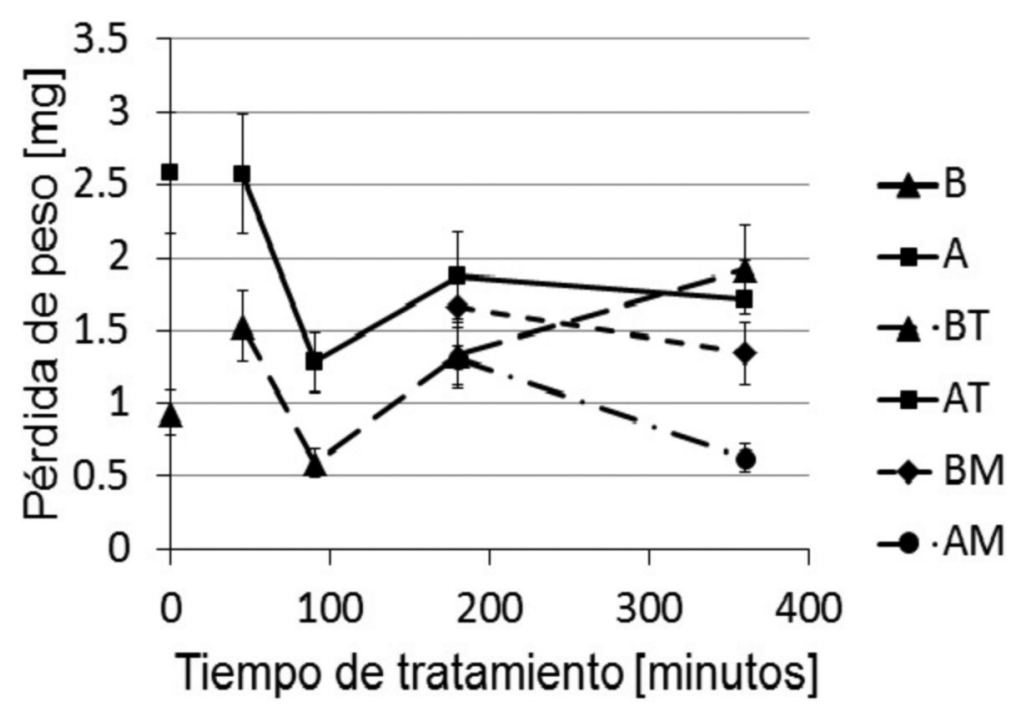

Figura 6. Comportamiento de las muestras bajo condiciones de desgaste por fricción.

Figure 6. Wear of specimens under conditions of dry friction.

mismo efecto enunciado para el caso del desgaste abrasivo. A diferencia del desgaste abrasivo, los mejores resultados para el tratamiento de mantenimiento se obtuvieron con la aleación con alto silicio. Esto está asociado a la mayor dureza que estas muestras presentan. En el caso del desgaste abrasivo lo determinante resulta ser la estructura de los carburos primarios.

En el caso de las muestras tratadas con austenización y mantenimiento, el mejor comportamiento ante la fricción seca de la aleación con bajo silicio resultó algo más marcado que en el caso del desgaste abrasivo. El comportamiento de estas muestras en función del tiempo de mantenimiento presenta un marcado valor mínimo para el tiempo de $90 \mathrm{~min}$, disminuyendo con rapidez la resistencia al desgaste para tiempos menores y mayores de este valor. Este comportamiento muestra que no es sólo la austenita la responsable del mismo, toda vez que las muestras con tiempos de mantenimiento superiores a los 90 min presentan similares volúmenes de esta fase y que el máximo volumen de austenita se alcanza a los $180 \mathrm{~min}$. La presencia del mínimo de desgaste para este tiempo de mantenimiento está asociada a la estructura más resistente, puesto que para ese tiempo la ferrita componente de la perlita mantiene contenidos de carbono y cromo relativamente elevados, debido a que la precipitación de carburos secundarios es aún incipiente. Para tiempos más largos la precipitación de carburos debilita la matriz y no ejerce un efecto importante sobre la resistencia al desgaste.
Este comportamiento también se aprecia en la aleación con alto contenido de silicio, pero menos marcado.

La magnitud de la fuerza media de fricción (Tabla II) se determinó teniendo en cuenta todo el tiempo de ensayo de la muestra, dado que, en algunas de ellas resultaba difícil la definición con exactitud de una zona de fricción estable. Dado el largo tiempo de ensayo utilizado (224 min) y la elevada frecuencia de muestreo (4 lecturas/s) la influencia de la zona inicial resulta pequeña y el valor obtenido es semejante al valor medio de la zona de fricción estable y permite comparar los resultados de las muestras sin errores apreciables.

Este valor de la fuerza media de fricción en el ensayo no muestra variaciones significativas para las diferentes muestras ensayadas lo que conduce a valores semejantes del coeficiente de fricción. No obstante se presentan algunas tendencias destacables. El valor medio de la fuerza de fricción en el grupo de las aleaciones con bajo silicio es ligeramente superior al valor medio del grupo de las aleaciones con alto silicio, aunque esta diferencia no supera la variabilidad presente.

La fuerza de fricción se mantiene sin variaciones notables a lo largo del ensayo, aunque con tendencia a aumentar en el tiempo, como se aprecia en la figura 7 , correspondiente a la muestra $\mathrm{A}$.

En algunas muestras, fundamentalmente las que presentan los mayores contenidos de austenita, se observa un incremento sostenido de la fuerza a lo largo del ensayo, como se muestra en las figuras 8 (Muestra AT180) y 9 (Muestra BT180). 


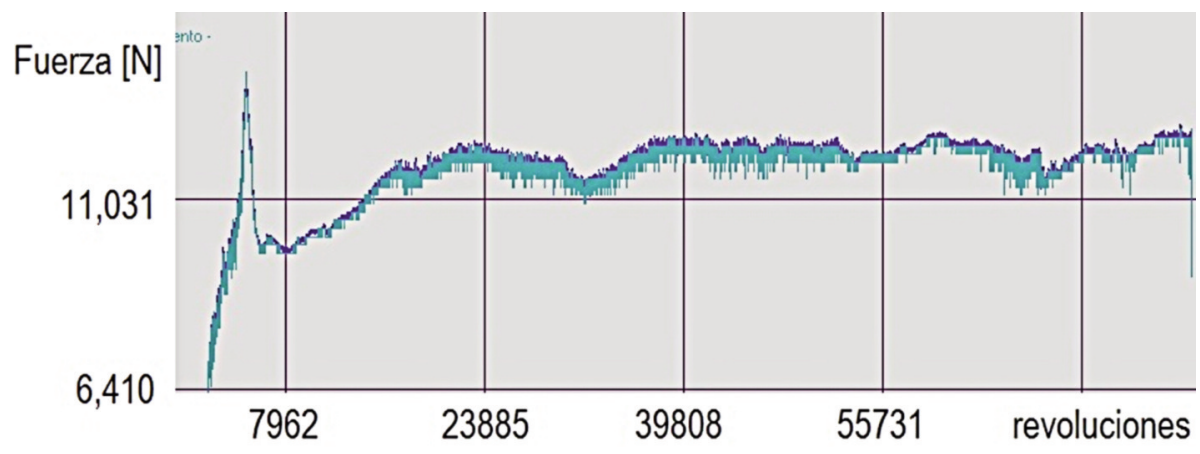

Figura 7. Evolución de la fuerza de fricción durante el ensayo. Muestra A.

Figure 7. Evolution of the frictional force during the test. Sample A.

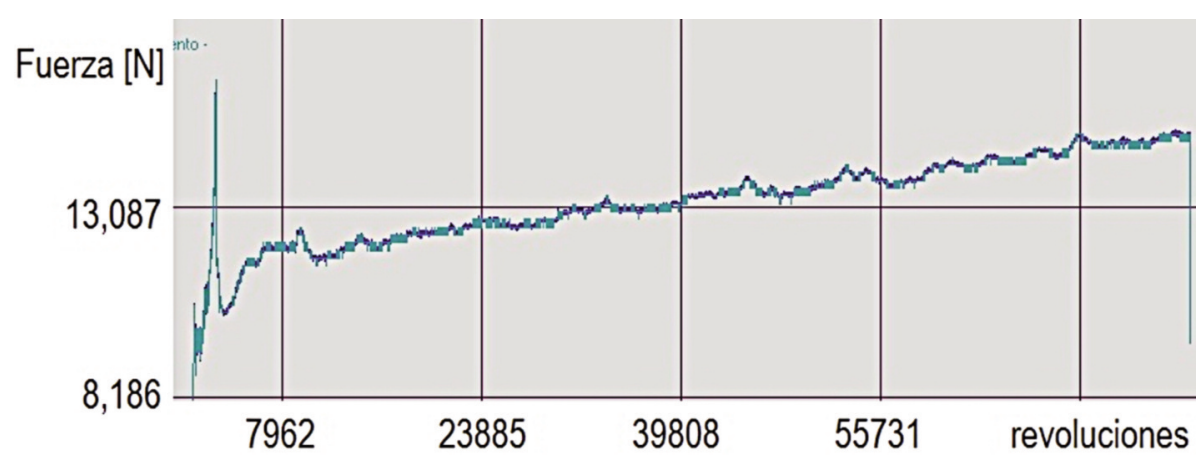

Figura 8. Evolución de la fuerza de fricción durante el ensayo. Muestra AT180.

Figure 8. Evolution of the frictional force during the test. Sample AT180.

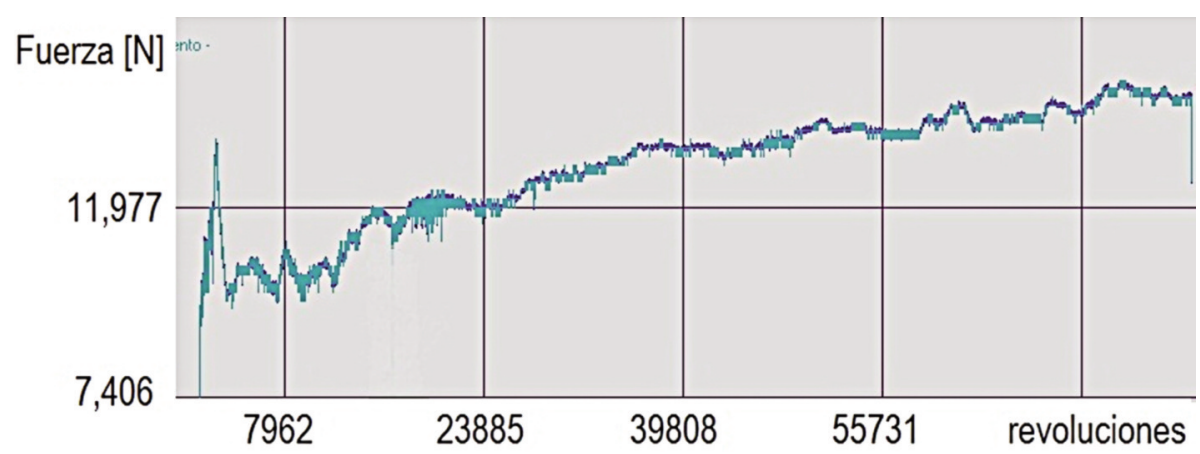

Figura 9. Evolución de la fuerza de fricción durante el ensayo. Muestra BT180.

Figure 9. Evolution of the frictional force during the test. Sample BT180.

Este incremento sostenido de la fuerza de fricción a lo largo del ensayo puede asociarse a la presencia de la austenita y su endurecimiento por la deformación. Sin embargo, otras muestras sin presencia de austenita en su microestructura presentan un incremento similar (Fig. 10, correspondiente a 


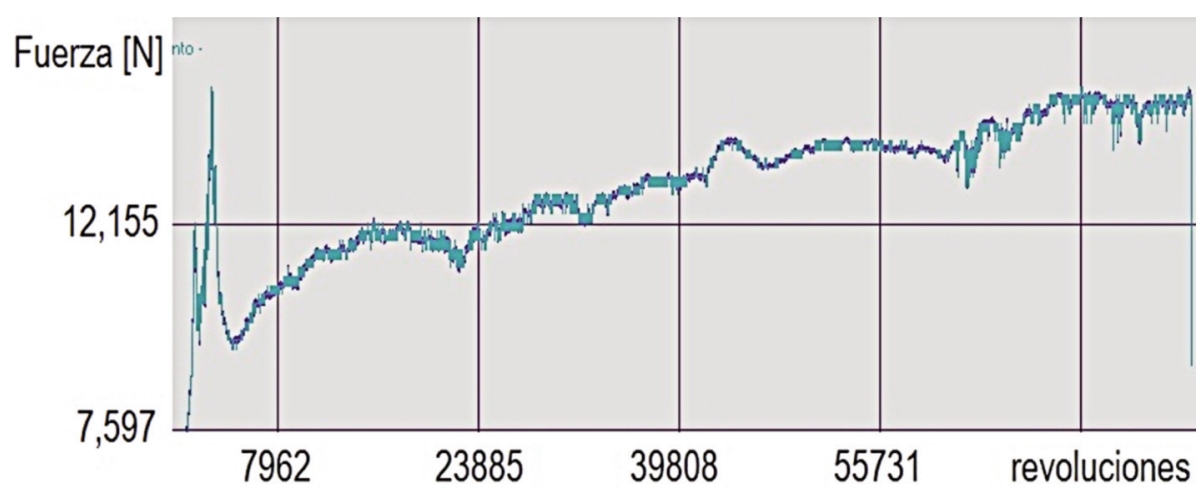

Figura 10. Evolución de la fuerza de fricción durante el ensayo. Muestra B.

Figure 10. Evolution of the frictional force during the test. Sample B.

la muestra B). Este comportamiento evidencia que existen otros factores influyentes, además del contenido de austenita, en los cuales es necesario profundizar.

\section{CONCLUSIONES}

- Los resultados obtenidos en el presente trabajo muestran algunos aspectos destacables con respecto a la influencia del silicio, en el marco de las condiciones de rápido enfriamiento aplicadas.

- Desde el punto de vista de la influencia sobre la estructura se aprecia que el aumento del contenido de silicio en las aleaciones estudiadas, para las condiciones de enfriamiento aplicadas, reduce el tamaño de los carburos primarios y mejora su distribución, logrando una estructura con carburos más pequeños y menor distancia entre ellos, lo que se manifiesta en sus propiedades.

- El contenido de cromo en matriz que se presenta bajo estas condiciones de enfriamiento es ligeramente superior al reportado para aleaciones semejantes coladas en arena. El incremento del contenido de silicio en las aleaciones estudiadas conduce a un ligero empobrecimiento en cromo tanto de la matriz como de los carburos primarios.

- La adición de silicio no mostró gran influencia sobre la dureza de las muestras en general. En estado bruto de colada no se aprecia diferencia. Para las muestras tratadas, no importa cuál de los tratamientos sea aplicado, la aleación con alto silicio se manifiesta con una dureza ligeramente superior. Dentro de las muestras estudiadas los mayores resultados de dureza corresponden a la aleación alta en silicio con el tratamiento subcrítico.

- Para la generalidad de los tratamientos aplicados la adición del silicio provoca muy poca disminución en la resistencia al desgaste, tanto abrasivo como para las condiciones de fricción seca. Se exceptúa de esta tendencia la aleación de alto silicio con tratamiento subcrítico en las condiciones de fricción seca. Para este tipo de desgaste la aleación con bajo silicio y tratamiento subcrítico fue la que mostró los mejores resultados tras un tiempo de $6 \mathrm{~h}$.

- Desde el punto de vista económico el tratamiento subcrítico resulta del mayor interés. Este tratamiento mostró buenos resultados ante la abrasión y la fricción seca, constituyendo en ambos casos una opción ventajosa.

- Resulta un elemento destacable que el estado bruto de colada constituye una opción satisfactoria para el caso del desgaste abrasivo, lo que califica a las condiciones de enfriamiento rápido como un factor de la mayor importancia en el logro de aleaciones resistentes al desgaste abrasivo. En este caso el contenido de silicio no mostró diferencias apreciables en el comportamiento.

- En el caso de la fricción seca el contenido de silicio influye desfavorablemente en el comportamiento de la aleación en estado bruto de colada.

- En general el tratamiento de austenización y mantenimiento aplicado no mejora la resistencia al desgaste abrasivo ni al desgaste por fricción seca con respecto a las aleaciones en estado bruto de colada por lo que no se justifica económicamente su aplicación con estos objetivos.

- En las probetas austenizadas el factor influyente fundamental en el comportamiento de la resistencia al desgaste abrasivo es la distribución de los carburos primarios. 
- La fuerza de fricción no presentó variaciones importantes ante la presencia del silicio o los diferentes tratamientos, aunque muestra tendencia a disminuir con el aumento del tiempo de mantenimiento para ambos tratamientos. Los menores valores se presentan en las aleaciones en estado bruto de colada.

- La presencia de austenita en las muestras se manifestó en un incremento de la fuerza de fricción con respecto al tiempo.

\section{REFERENCIAS}

[1] H. S. Avery, Materials for the mining industry, Climax Molybdenum, Vail, Col., 1974.

[2] J. Dodd y J. L. Parks, Factors affecting the production and performance of thick section high $\mathrm{Cr}$ Mo alloy iron castings, Climax Molybdenum, Greenwich,Conn., 1980.

[3] G. Laird, Trans. AFS 101 (1993) 497-503.

[4] F. Maratray, Bulletin du Cercle dÉtudes des Métaux Novembre (1979) 11-54.

[5] J. L. Parks, Characteristics of as-cast and subcritically heat-treated high $\mathrm{Cr}$-Mo white Irons of thick section castings, Climax Molybdenum, Vail,Col., 1978.

[6] I. Fernández-Pariente y F. J. Belzunce-Varela, Rev. Metal. Madrid 42 (2006) 279-286.

[7] K. A. Kibble y T. H. Pearce, Cast Metals 6 (1993) 9-15.

[8] R. L. Pattyn, Trans. AFS 101 (1993) 161-167.

[9] K. K. Singh, R. S. Verma y G. M. D. Murty, J. Mater.Eng. Perform. 18 (2009) 438-440.

[10] R. B. Gundlach, Trans. AFS 82 (1974) 309-316.

[11] F. Maratray y R. Usseglio-Nanot, Atlas Transformation characteristics of $\mathrm{Cr}$ and $\mathrm{Cr}-\mathrm{Mo}$ white irons, Climax Molybdenum, Belgium, 1970, p. 198.

[12] Z. Sun, R. Zuo, C. Li, B. Shen, J. Yan y S. Huang, Mater. Charact. 53 (2004) 403-409.
[13] A. Bedolla-Jacuinde, L. Arias y B. Hernández, J. Mater. Eng. Perform. 12 (2003) 371-382.

[14] J. Asensio, J. A. Pero-Sanz y J. I. Verdeja, Mater. Charact. 49 (2003) 83- 93.

[15] K. H. Zum-Gahr y G. T. Eldis, Wear 64 (1980) 175-194.

[16] O. N. Dogan y J. A. Hawk, Trans. AFS 105 (1997) $167-174$.

[17] O. N. Dogan y J. A. Hawk, Wear 189 (1995) 136-142.

[18] C. Cetinkaya, Mater. Design 27 (2006) 437-445.

[19] W. W. Cias, Trans. AFS 82 (1974) 317-328.

[20] L. Wei, J. lron Steel Res. Int. 14(3) (2007) 47-50.

[21] A. Bedolla-Jacuinde y W. M. Rainforth, Wear 250 (2001) 449-461.

[22] H. Qing-yu, H. Zhen-yi y W. Jing-tao, J. Iron Steel Res. Int. 16(4) (2009) 33-38.

[23] A. E. Karantzalis, A. Lekatou y H. Mavros, J. Mater. Eng. Perform. 18 (2009) 174-181.

[24] I. Morales, W. Hormaza y L. Méndez, Revista de Ingeniería de la Universidad de los Andes. Bogotá, Colombia. 30 (2009).

[25] R. L. Pattyn, Trans. AFS 102 (1994) 107-115.

[26] J. Wang, R. L. Zuo, Z. P. Sun, C. Li, H. H. Liu, H. S. Yang, B. L. Shen y S. J. Huang, Mater. Charact. 55 (2005) 234- 240.

[27] J. Wang, C. Li, H. H. Liu, H. S. Yang, B. L. Shen, S. Gao y S. J. Huang, Mater. Charact. 56 (2006) 73-78.

[28] M. J. Santofimia, Tesis Doctoral, Dpto. de Física de Materiales, vol PhD, Univ. Complutense de Madrid, 2006.

[29] R. S. Jackson, JISI 208 (1970) 163-167.

[30] F. Maratray y R. Usseglio-Nanot (Eds.), Factors affecting the structure of chromium and chromiummolybdenum white irons, Climax Molybdenum S.A., Paris,France, 1970, p. 32.

[31] A. Kulmburg, Bulletin du Cercle d'ètudes des mètaux Novembre (1973) 475-490.

[32] L. Ferreiro, Tesis Doctoral, Dpto. de Ing. Industrial II, Universidade da Coruña, Ferrol, 2011. 